\section{SP3-86 VALIDATION OF A SINGLE QUESTION FOR QUALITY OF LIFE ASSESSMENT IN CHILEAN OLDER PEOPLE}

doi:10.1136/jech.2011.1429760.86

L Lera, ${ }^{*}$ C Albala, H Sanchez, A Fuentes, A Barbara, X Cea. INTA/University of Chile, Santiago, Chile

Aim To validate a single question of self-reported quality of life as a reliable instrument in population studies.

Method Cross sectional validation of a single question about $\mathrm{QoL}$ against the quality of life SF-36 (Short-Form 36 Health Survey). The study was performed in a national representative sample of 4762 community-dwelling people 61-101 y (38.8\% men) from a national study on dependency done in Chile in 2009-2010. All subjects completed household interviews (face to face), including the question "How is your quality of life?" (Excellent, Good, Fair, Poor and Very Poor). To validate it we used the summary measures mental and physical health of the SF-36, estimated with Chilean-Specific scoring algorithms previously validated. The relation of summarised score to each category was studied using Kruskal-Wallis test and the non-parametric test for trend across ordered groups.

Results The mean score (using survey design) of the mental and physical components follow a gradient from Excellent, Good, Fair, Poor to Very Poor being the higher score excellent and the lower very poor. Mental component mean scores: 56.7, 54.4, 49.0, 39.1, 37.6; physical component mean scores: 54.1, 51.4, 46.5, 41.1, 38.1 $(p<0.0001)$ Similar results were obtained in both sexes.

Conclusion The single question about $\mathrm{QoL}$ is a reliable measure, easy to interpret and barely time consuming. For this reason appears as an excellent instrument to be used in large population studies.

\section{SP3-87 CHARACTERISATION OF SENTINEL SURVEILLANCE OF INFLUENZA-LIKE ILLNESS (ILI) AND HOSPITAL SURVEILLANCE OF SEVERE ACUTE RESPIRATORY INFECTIONS (SARI) IN PARAGUAY}

doi:10.1136/jech.2011.1429760.87

\begin{abstract}
${ }^{1} \mathrm{M}$ V Horch, ${ }^{*} 1,2 \mathrm{~A}$ Cabello, ${ }^{3} \mathrm{~L}$ Bobadilla, ${ }^{3} \mathrm{C}$ Vazquez, ${ }^{1,2} \mathrm{M}$ Samudio, ${ }^{1} \mathrm{I}$ Allende. ${ }^{1}$ Dirección General de Vigilancia de la Salud (DGVS), Asuncion, Paraguay; ${ }^{2}$ Instituto de Invstigaciones en ciencias de la salud, Asuncion, Paraguay; ${ }^{3}$ Laboratorio Central de Salud Publica, Asuncion, Paraguay
\end{abstract}

In 2010, based on the experience gained in the 2009 influenza pandemic, the surveillance is strengthened with the implementation of the hospital surveillance of SARI.

Objective To determine the epidemiological characteristics and etiological agents of ILI and SARI.

Methodology All cases from sentinel sites in the period from January to October 2010 were included. The analysed variables were: hospitalisations by epidemiological week, age, sex, entrance to ICU, death, presence of comorbidity, risk factors, and identification of the causative agent.

Results Sentinel Surveillance of ILI detected 799 patients. The most affected age group was young adults (15-39 years of age) with $55.82 \%(446 / 799)$. Only $11 \%$ (83/781) had some co morbidity and/ or risk factor. Regarding SARI hospital surveillance, 1692 notifications were recorded. Of these, $10.17 \%(172 / 1692)$ required treatment in intensive care unit (ICU), 53 patients died (specific lethality for SARI $3.13 \%$ ). The distribution by age groups showed an increased risk in children under 5 and older than 60 , with rates of $51 \%$ (868/ $1692)$ and $16 \%(274 / 1692)$, respectively. Nearly $30 \%(479 / 1692)$ of patients had comorbidity and/or risk factors. Samples were taken in $71.3 \%$ of cases $(1206 / 1692)$ and positivity was $22.6 \%(273 / 1206)$. The most frequently identified viruses were RSV, 39\% (106/273), Influenza A H3, 18\% (49/273) and influenza A H1N1, 15\% (41/273).
Conclusions With the incorporation of the SARI surveillance, respiratory viruses have been better characterised. The Influenza A H1N1 virus had a similar pattern to the seasonal respiratory viruses.

\section{SP3-88 SOCIAL DETERMINANTS OF SUBFERTILITY IN WOMEN WITH A SUCCESSFUL PREGNANCY}

doi:10.1136/jech.2011.142976o.88

C Pereira, ${ }^{*}$ E Coutinho, A Silva, J Duarte, N Veiga, C Chaves, M Ferreira, P Nelas, E Carril. CI\&DETS, Health School, Polytechnic Institute of Viseu, Viseu, Portugal

Introduction Socioeconomic conditions are rarely considered in the epidemiology of subfertility. This study determined the effect of current and past socioeconomic conditions on the occurrence of subfertility in women with a successful pregnancy.

Methods Women were recruited in 2005/6 for Geração XXI, a Portuguese birth cohort. Personal interviews were conducted to collect socio-demographic and pregnancy-related data. Life-time subfertility was self-reported and defined as trying to conceive for more than 1 year with no success. Education, income, working condition and marital status were proxy indicators of social class. The childhood circumstances of the women were accessed by parents' education and amenities at age 12. The analysis considered 7916 mothers. Logistic regression models were fitted to estimate the odds (OR) of being sub-fertile according socioeconomic circumstances, stratified by number of previous pregnancies ( 1 vs $>1)$ and age ( $<30$ vs $\geq 30$ years).

Results Sub-fertility was reported by $9.1 \%$ of the women $(n=719)$ Prevalence was $8.7 \%$ among primigravidae and $9.5 \%$ in multigravidae ( $84 \%$ of the multigravidae were multiparous). Almost $2 / 3$ sought medical help. Among young primigravidae, sub-fertility decreased with education ( $>12$ vs $\leq 6$ years: $O R=0.2095 \%$ CI 0.11 to 0.39 ), and was lower among single women $(\mathrm{OR}=0.2795 \% \mathrm{Cl}$ 0.12 to 0.59 ) and smokers (OR $=0.60 ; 95 \%$ CI 0.40 to 0.94$)$. Only education showed a significant association with sub-fertility in older primigravidae. Within multigravidae higher education levels increased the odds. Maternal childhood conditions were no longer significant after adjustment for current social circumstances.

Conclusions Age and the number of previous births modified the effect of social conditions on sub-fertility which was stronger among younger and primigravidae women.

\section{SP3-89 SOCIAL DETERMINANTS OF SUBFERTILITY IN WOMEN WITH A SUCCESSFUL PREGNANCY}

doi:10.1136/jech.2011.142976o.89

${ }^{1,2} \mathrm{~S}$ Correia, ${ }^{* 1,2} \mathrm{~T}$ Rodrigues, ${ }^{1,2} \mathrm{H}$ Barros. ${ }^{1}$ Department of Hygiene and Epidemiology, University of Porto Medical School, Porto, Portugal; ${ }^{2}$ Institute of Public Health University of Porto, Porto, Portugal

Introduction Socioeconomic conditions are rarely considered in the epidemiology of subfertility.

Objectives Access the effect of current and past socioeconomic conditions on the occurrence of subfertility in women with a successful pregnancy.

Methods Women were recruited in 2005/6 for Geração XXI, a Portuguese birth cohort. Personal interviews were conducted to collect socio-demographic and pregnancy-related data. Life-time subfertility was self-reported and defined as trying to conceive for more than 1 year with no success. Education, income, working condition and marital status were proxy indicators of social class. The childhood circumstances of the women were accessed by parents' education and amenities at age 12. The analysis considered 7916 mothers. Logistic regression models were fitted to estimate the odds (OR) of being subfertile according socioeconomic circumstances, stratified by number of previous pregnancies ( $1 \mathrm{vs}>1$ ) and age ( $<30 \mathrm{vs} \geq 30$ years). 